\title{
Stress Analysis of a Landing Gear Using the Davies Method
}

\author{
Gonzalo Guillermo Moreno ${ }^{1 \star}$, César Augusto Peña1, Oscar Eduardo Gualdron¹ \\ ${ }^{1}$ Department of Mechanical Engineering, University of Pamplona, Norte de Santander, Pamplona, Route to Bucaramanga Km 1, \\ Colombia \\ * Corresponding author, e-mail: gmoren@hotmail.com
}

Received: 06 May 2020, Accepted: 20 May 2020, Published online: 01 July 2020

\begin{abstract}
Structure and mechanism analysis often involves the use of advanced simulation programs, which are not always available to users. The Davies method has proven to be an efficient and easily applied tool in this type of analysis, allowing the obtaining of the stresses present in the links and the necessary torque to keep the device in optimal operative conditions. All these standards will set the foundations for the development and proper design of the mechanism. Taking into account the afore mentioned, this article describes in detail the use and applications of the Davies method for stress and kinematic analysis of a landing gear.
\end{abstract}

Keywords

landing gear, Davies method, stress analysis, mechanical design

\section{Introduction}

Stress and deformities are essential criteria while designing the components for mechanisms. Due to this, it is important to determine the maximum forces that act upon them. Furthermore, these analyses also allow setting the maximum torques that the power elements of the mechanism must bear $[1,2]$.

There are several design programs nowadays, but in some cases, the costs related to their implementation make it impossible for some companies to carry out these analyses.

Taking these factors into account, the Davies method has proven to be a quite versatile tool, which allows countless analyses, among which are work capacity and strength in robots [3-5], stability analysis he heavy-duty vehicles [6-8], or mechanism optimization [9], among others.

For its implementation, the Davies method employs the screw theory, the graph theory, and the Kirchhoff laws. All of these theories allow setting all the equations that determine the kinematics and statics of mechanisms [10-13].

To demonstrate this theory, in this work, the stress and kinematics analysis of a landing gear subjected to extreme working loads is performed [14]. In Section 2, the implementation of the Davies method is detailed; afterward, in Section 3 the selected mechanism is analyzed, and the obtained results are discussed; and finally, conclusions are drawn in Section 4.

\section{Davies method}

The Davies method provides a systematic way to relate the joint forces and moments in closed kinematic chains [10]. This method is based on graph theory, screw theory, and the Kirchhoff cut-set law and it can be used to obtain the statics of a mechanism as a matrix expression [10]. The Davies method for static analysis can be briefly described through the following steps:

- Draw the kinematic chain of a given mechanism, identifying all of its " $n$ " links, " $f$ " external forces, and " $j$ " direct couplings (joints).

- Draw the direct coupling graph "GA" for the mechanism using the links of the mechanism as vertices, and the joints and external forces as edges. Assign positive directions to each edge with an arrow pointing from the minor to the major vertex.

- Write the incidence matrix of the direct coupling graph $[I]_{n, j+f}$.

- Generate the cut-set matrix $[Q]_{k, j+f}$ from $[I]_{n, j+f}$ using the Gauss-Jordan elimination method, where the number of cuts $(k=n-1)$ (identity matrix), and the number of chords $(l=j+f-n+1)$ are defined and depicted in the action graph.

- Write the expanded cut-set matrix $[Q]_{k ; c}$, where $c$ is the number of constraints of the joints and external forces of the mechanism.

- Write a wrench $\$ J_{\lambda ; c}$ for each constraint and external force of the mechanism, as follows (Eq. (1)): 
$\$_{J_{\lambda, c}}=\left[\begin{array}{l}0 \\ z \\ -y \\ 1 \\ 0 \\ 0\end{array}\right] J_{F x}+\left[\begin{array}{l}-z \\ 0 \\ x \\ 0 \\ 1 \\ 0\end{array}\right] J_{F y}+\left[\begin{array}{l}y \\ -x \\ 0 \\ 0 \\ 0 \\ 1\end{array}\right] J_{F z}+\left[\begin{array}{l}0 \\ 0 \\ 0 \\ 1 \\ 0 \\ 0\end{array}\right] J_{M x}+\left[\begin{array}{l}0 \\ 0 \\ 0 \\ 0 \\ 1 \\ 0\end{array}\right] J_{M y}+\left[\begin{array}{l}0 \\ 0 \\ 0 \\ 0 \\ 0 \\ 1\end{array}\right] J_{M z}$

where $\lambda$ is the degrees of freedom of the space in which the mechanism is intended to function.

- Replace each wrench $\$ J_{\lambda ; c}$ in the expanded cut-set matrix $[Q]_{k ; c}$ to obtain the generalized action matrix $\left[A_{N}\right]_{\lambda k ; c}$.

- Operate algebraically the generalized action matrix $\left[A_{N}\right]_{\lambda k ; c}$ to solve the system.

\section{Case study}

The mechanism of Fig. 1 is used to lower and retract the landing gear on small airplanes. If there are up to two different components of forces acting on the tire-ground interface $\left(P_{x}\right.$ - traction or brake force, and $P_{y}$ - normal force); determine the normal forces $\left(F_{n}\right)$ and the shear forces $\left(F_{p}\right)$ acting on the links, and the torque $\left(T_{x}\right)$ required to drive the link and to maintain the position of the mechanism [14], take into account that when the traction force is applied, the small shear deformation of the tire, it changes the point of application of the forces [15].

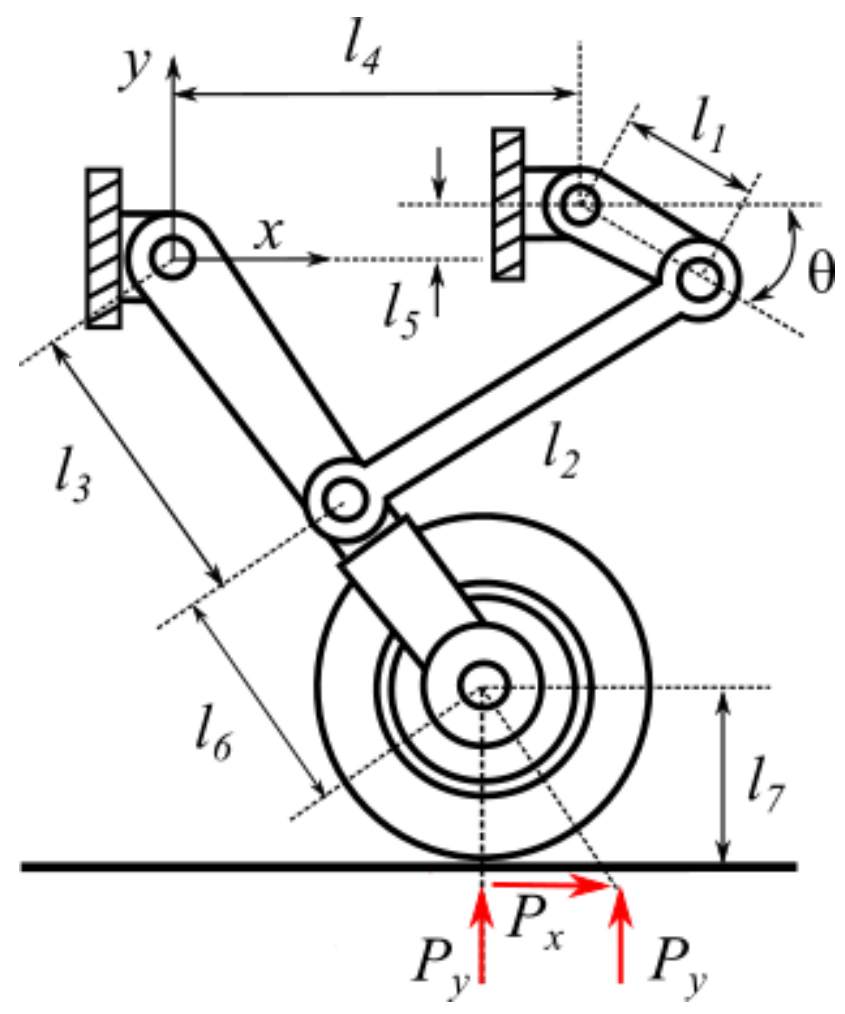

Fig. 1 Mechanism example 2.
Using the Extended Passive Joints Method (EPJM) [15], the mechanism (Fig. 1) can be represented by the kinematic chains shown in Fig. 2 (a). For this development, it was assumed that the tire has a radial deformation and a small shear deformation [15] due to the operation of the mechanism, and at the intermediate points of the links, prismatic joints were placed to determine the forces to which they are subjected.

Then, the kinematic chain of the mechanism is composed of ten links identified by letters $A$ (the base), $B, C$ and $H$ (link and damping system), $D$ and $E$ (link), $F$ and $G$ (link), and $I$ and $J$ (tire); and the nine joints are identified by numbers as follow: five revolute joints " $R$ " $(1,3,5$, 7 and 9), and five prismatic joints " $P$ " (2, 4, 6, 8 and 10), as shown in Figs. 2 (a) and (b).

The proposed mechanism has a passive actuator in each prismatic joint and an active actuator in the joint $3\left(T_{x}\right)$; these actuators control the movement of the mechanism.

Using graph theory, the mechanism of Fig. 2 (a) can be represented by the graph shown in Fig. 3 (a); where the vertices correspond to the links and the edges correspond to the constraints of each joint $[16,17]$ as well as the external forces present in the mechanism, this graph can also be represented by the incidence matrix $[I]_{10 \times 12}$ (Eq. (2)), additionally, solving Eq. (2) using the GaussJordan elimination method, the incidence matrix provides the cut set matrix $[Q]_{9 \times 12}$ of the mechanism, where each

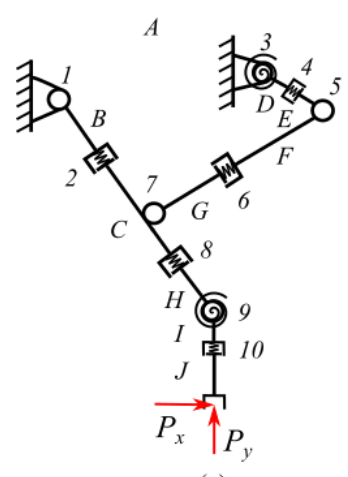

(a)

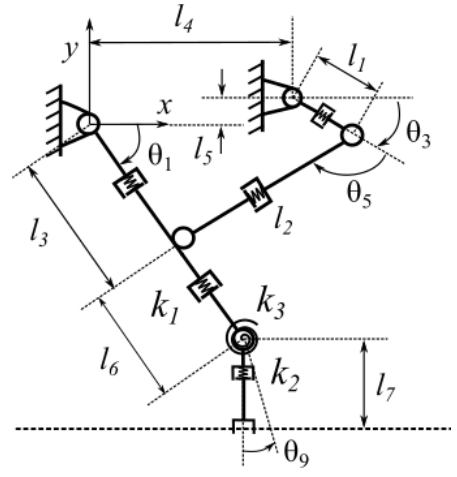

(b)
Fig. 2 (a) Kinematic chain of the mechanism. (b) Parameters of the mechanism. 


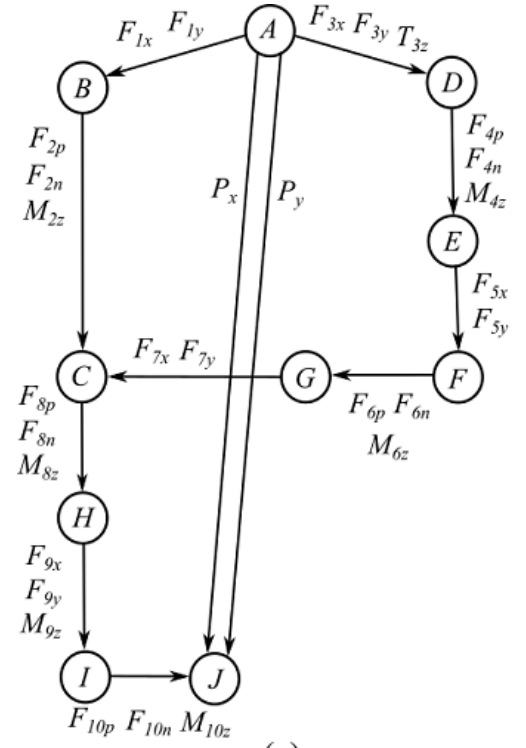

(a)

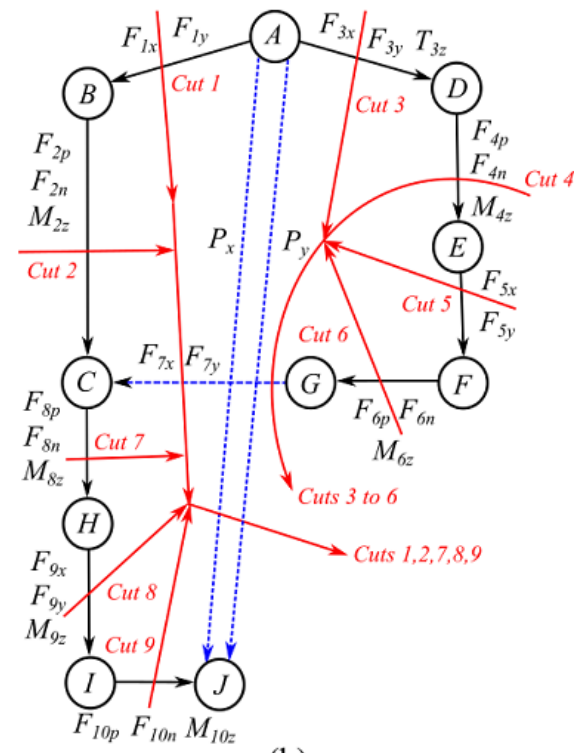

(b)

Fig. 3 (a) Direct coupling graph. (b) Cut-set action graph.

line represents a cut of the graph and the columns represent the joint and the external forces (Eq. (3)), which was reorganized, to identify nine (9) branches (edges 1-6 and 8-10 - identity matrix) and three (3) chords (edges 7, $P_{x}$, and $P_{y}$ ), as shown in Fig. 3 (b).

Furthermore, in Fig. 3 (b) all of the constraints present in the joints are represented as edges, which allows the amplification of the cut-set matrix (Appendix).
In this context, revolute joints (1, 3, 5, 7 and 9) have constraints in the $x$ and $y$ axes, and prismatic joints $(2,4,6$, 8 and 10) have constraints in their axis of movement (normal forces $\left(F_{n}\right)$ ), perpendicular their axis of movement (shear forces $\left(F_{p}\right)$ ), and moment in the $x$-axis.

Considering a static analysis in two-dimensional space [10], all the wrenches of the mechanism together comprise the action matrix $\left[A_{d}\right]$ given by Eq. (4).

$$
\begin{aligned}
& \begin{array}{llllllllllll}
1 & 2 & 3 & 4 & 5 & 6 & 7 & 8 & 9 & 10 & P_{x} & P_{y}
\end{array} \\
& A\left[\begin{array}{cccccccccccc}
1 & 0 & 1 & 0 & 0 & 0 & 0 & 0 & 0 & 0 & 1 & 1 \\
-1 & 1 & 0 & 0 & 0 & 0 & 0 & 0 & 0 & 0 & 0 & 0
\end{array}\right] \\
& C \quad \begin{array}{cccccccccccc}
C & -1 & 0 & 0 & 0 & 0 & -1 & 1 & 0 & 0 & 0 & 0
\end{array} \\
& \begin{array}{c|cccccccccccc}
D & 0 & 0 & -1 & 1 & 0 & 0 & 0 & 0 & 0 & 0 & 0 & 0
\end{array} \\
& {[I]_{10 \times 12}=E\left[\begin{array}{lllllllllllll} 
& 0 & 0 & 0 & -1 & 1 & 0 & 0 & 0 & 0 & 0 & 0 & 0
\end{array}\right.} \\
& F=0 \begin{array}{llllllllllll}
F & 0 & 0 & 0 & -1 & 1 & 0 & 0 & 0 & 0 & 0 & 0
\end{array}
\end{aligned}
$$

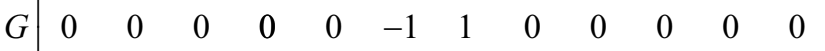

$$
\begin{aligned}
& \begin{array}{r|rlllllllllll}
H & 0 & 0 & 0 & 0 & 0 & 0 & 0 & -1 & 1 & 0 & 0 & 0
\end{array} \\
& \begin{array}{llllllllllllll}
I & 0 & 0 & 0 & 0 & 0 & 0 & 0 & 0 & -1 & 1 & 0 & 0
\end{array} \\
& J\left[\begin{array}{llllllllllll}
0 & 0 & 0 & 0 & 0 & 0 & 0 & 0 & 0 & -1 & -1 & -1
\end{array}\right] \\
& \begin{array}{llllllllllll}
1 & 2 & 3 & 4 & 5 & 6 & 8 & 9 & 10 & 7 & P_{x} & P_{y}
\end{array} \\
& \text { Cut } 1\left[\begin{array}{llllllllllll}
1 & 0 & 0 & 0 & 0 & 0 & 0 & 0 & 0 & 1 & 1 & 1
\end{array}\right] \\
& \text { Cut } 20 \begin{array}{llllllllllll}
0 & 1 & 0 & 0 & 0 & 0 & 0 & 0 & 0 & 1 & 1 & 1
\end{array}
\end{aligned}
$$

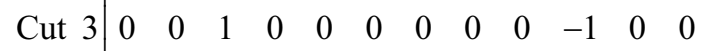

$$
\begin{aligned}
& {[Q]_{9 \times 12}=\begin{array}{l|llllllllllll}
\text { Cut } 4 & 0 & 0 & 0 & 1 & 0 & 0 & 0 & 0 & 0 & -1 & 0 & 0 \\
\text { Cut } 5 & 0 & 0 & 0 & 0 & 1 & 0 & 0 & 0 & 0 & -1 & 0 & 0
\end{array}} \\
& \text { Cut } 60 \begin{array}{llllllllllll}
0 & 0 & 0 & 0 & 0 & 1 & 0 & 0 & 0 & -1 & 0 & 0
\end{array} \\
& \text { Cut } 70 \begin{array}{llllllllllll}
0 & 0 & 0 & 0 & 0 & 0 & 1 & 0 & 0 & 0 & 1 & 1
\end{array} \\
& \text { Cut } 8 \quad 0 \begin{array}{llllllllllll}
0 & 0 & 0 & 0 & 0 & 0 & 1 & 0 & 0 & 1 & 1
\end{array} \\
& \text { Cut } 9\left[\begin{array}{llllllllllll}
0 & 0 & 0 & 0 & 0 & 0 & 0 & 0 & 1 & 1 & 1 & 1
\end{array}\right]
\end{aligned}
$$




$$
\left[A_{d}\right]_{3 \times 29}=\left[\begin{array}{lllllll}
\$_{F_{1 x}}^{A} & \$_{F_{1 y}}^{A} & \$_{F_{2 p}}^{A} & \cdots & \$_{T_{3 z}}^{A} & \$_{P_{x}}^{A} & \$_{P_{y}}^{A}
\end{array}\right]
$$

The wrench can be represented by a normalized wrench and its magnitude. Therefore, from the Eq. (4) the unit action matrix and the magnitude action vector are obtained, as represented by Eqs. (5) and (6).

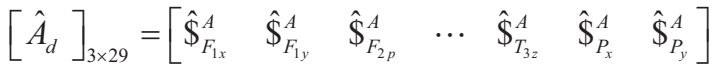

$$
\begin{aligned}
& {[\Psi]_{29 \times 1}=\left[\begin{array}{lllllll}
F_{1 x} & F_{1 y} & F_{2 p} & \cdots & T_{3 z} & P_{x} & P_{y}
\end{array}\right]}
\end{aligned}
$$

Using the cut-set law [18-20], the algebraic sum of the normalized wrenches, Eqs. (6) and (7), that belong to the same cut (Fig. 3 (b) and Appendix) must be equal to zero. Therefore, the statics of the mechanism can be defined, as exemplified in Eq. (7):

$\left[\hat{A}_{n}\right]_{27 \times 29}[\Psi]_{29 \times 1}^{T}=[0]_{27 \times 1}$.

In Eq. (7) is necessary to identify the set of primary variables $\left[\Psi_{p}\right]$ (known variables), among the variables of $\Psi$. Once identified, the system is divided into two sets, as shown in Eq. (8),

$\left[\hat{A}_{n s}\right]_{27 \times 27}\left[\Psi_{s}\right]_{27 \times 1}^{T}+\left[\hat{A}_{n p}\right]_{27 \times 2}\left[\Psi_{p}\right]_{2 \times 1}^{T}=[0]_{27 \times 1}$

where $\left[\Psi_{p}\right]$ is the primary variable vector, $\left[\Psi_{s}\right]$ is the second variable vector (unknown variables), $\left[\hat{A}_{n p}\right]$ are the columns corresponding to the primary variables and $\left[\hat{A}_{n s}\right]$ are the columns corresponding to the secondary variables.

In this case, the primary variables vector is (Eq. (9)):

$\left[\Psi_{p}\right]_{2 \times 1}=\left[\begin{array}{ll}P_{x} & P_{y}\end{array}\right]^{T}$.

Solving the system in Eq. (8) using the Gauss-Jordan elimination method, all secondary variables of the system being functions of the primary variables of the mechanism $\left(P_{x}\right.$ and $\left.P_{y}\right)$.

Taking into account this development and the parameters of the Table 1, for the simulation, the airplane's traction force was gradually increased, which changed the force application point, due to its deformation [15].

Taking these aspects into account, in Fig. 4, it can be observed that the greater normal force in the mechanism is compression, which is present on the $G F$ link $\left(F_{6 n}\right)$.

Similarly, it can be observed that the highest shear force is present on the body of the tire $\left(F_{10 p}\right)$ and the $D E \operatorname{link}\left(F_{4 p}\right)$.

The forces present on the links (Figs. 4 and 5), can be combined to determine the Von Mises stress (Fig. 6) based on the link area (Eq. (10)), these criteria allow an optimal design of the links in both their setting and the material chosen for them.
Table 1 Parameters of the example.

\begin{tabular}{lcc}
\hline Parameters & Inputs & Units \\
\hline$l_{1}$ & 0.54 & $\mathrm{~m}$ \\
$l_{2}$ & 0.91 & $\mathrm{~m}$ \\
$l_{3}$ & 0.71 & $\mathrm{~m}$ \\
$l_{4}$ & 0.61 & $\mathrm{~m}$ \\
$l_{5}$ & 0.23 & $\mathrm{~m}$ \\
$l_{6}$ & 0.61 & $\mathrm{~m}$ \\
$l_{7}$ & 0.54 & $\mathrm{~m}$ \\
$\theta_{3}$ & 30 & $\mathrm{degree}$ \\
$k_{1}$ & 220 & $\mathrm{kN} \mathrm{m}^{-1}$ \\
$k_{2}$ & 160 & $\mathrm{kN} \mathrm{m}^{-1}$ \\
$k_{3}$ & 25 & $\mathrm{kN} \mathrm{m} \mathrm{rad}^{-1}$ \\
$P_{x}$ & 3 & $\mathrm{kN}_{y}$ \\
$P_{y}$ & 9.8 & $\mathrm{kN}$
\end{tabular}

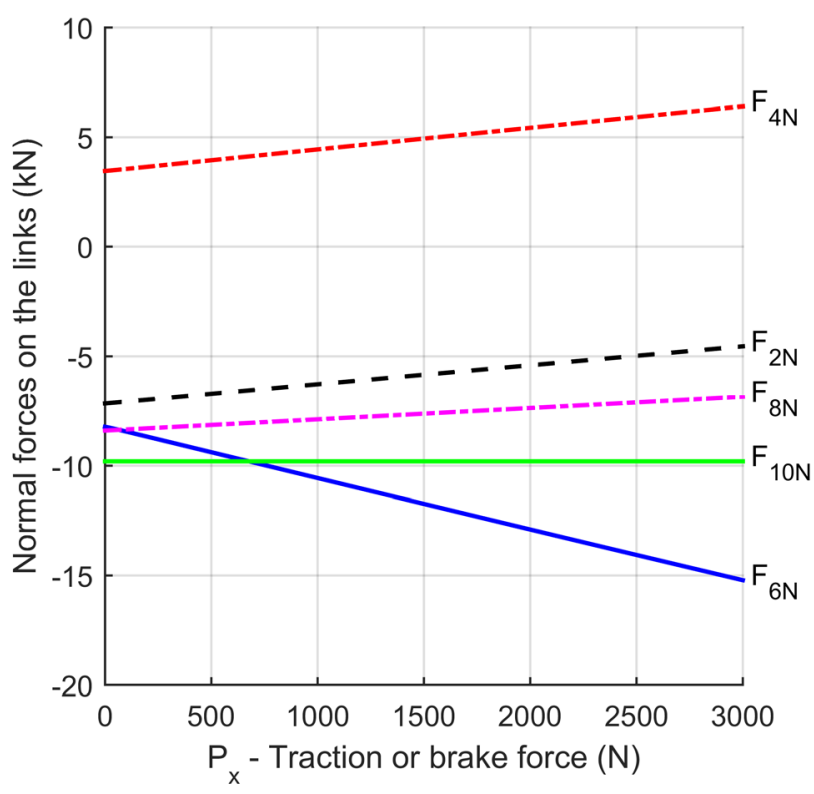

Fig. 4 Normal forces.

$\sigma_{\mathrm{VM}}=\frac{1}{A} \sqrt{F_{n}^{2}+3 F_{P}^{2}}$

Additionally, in Fig. 7 it can be observed how the increase of the traction force of the airplane raises the torque required to keep the taking off or landing mechanism working.

\section{Conclusions}

The technique applied allows determining systemically the forces that act upon the mechanisms and on the elements that compose them, which is quite essential since, using these values, a design and optimal selection of elements and equipment that compose a mechanism can be done.

The application of traction force makes a shear deformity appear in the tire, making a point of application of 


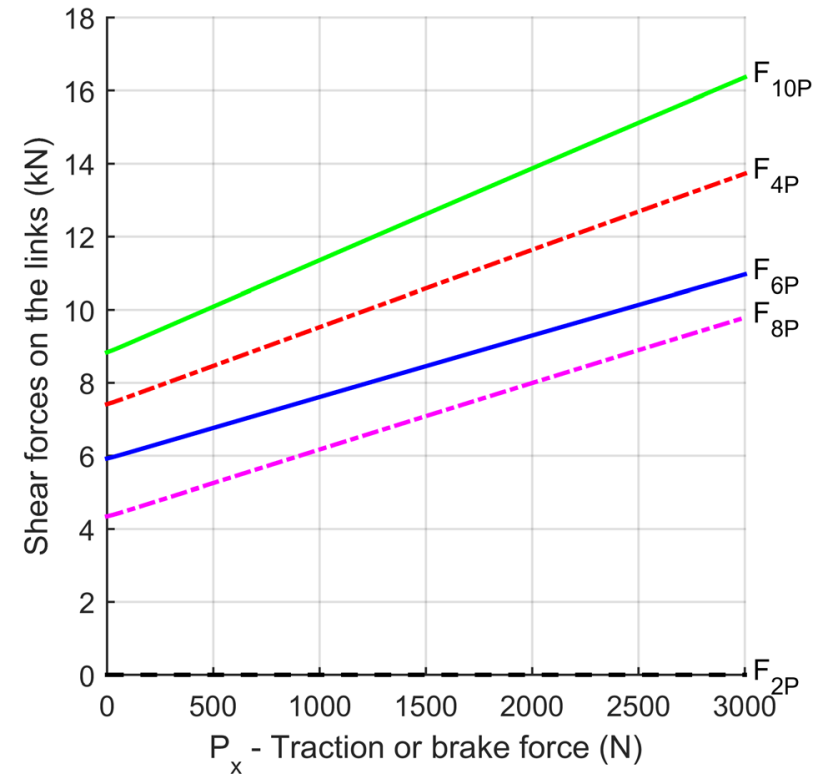

Fig. 5 Shear forces.

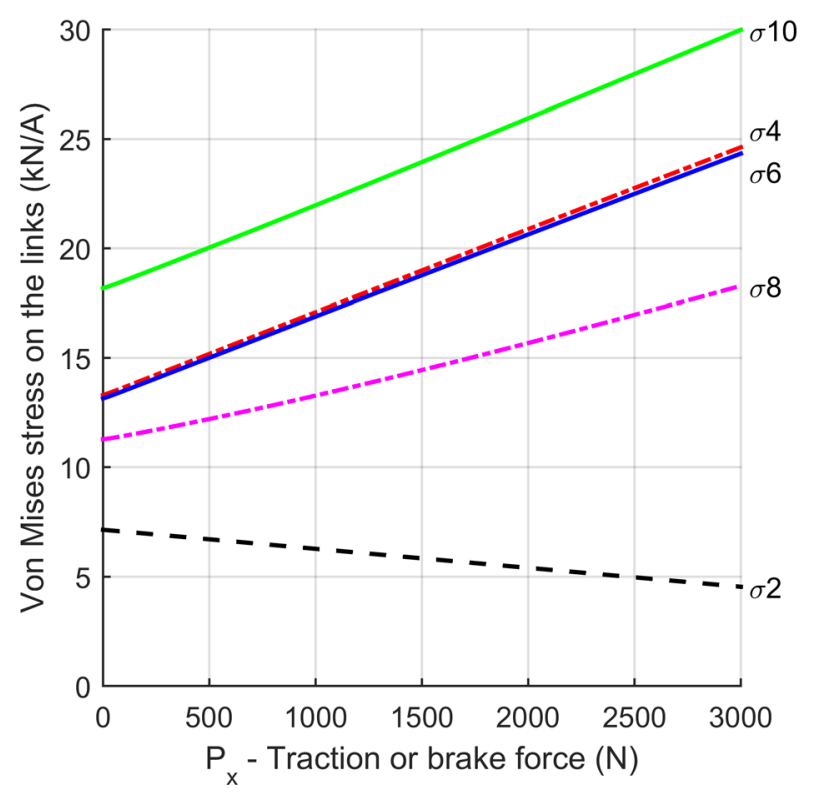

Fig. 6 Von Mises stress.

\section{References}

[1] Mejia Rincon, L. "Wrench capability of planar manipulators", PhD Thesis, Federal University of Santa Catarina, 2016.

[2] Contreras, M., Gonzalo, G. "A kinestatic model for the three-dimensional static analysis of long combination vehicles", PhD Thesis, Federal University of Santa Catarina, 2017.

[3] Frantz, J. C., Mejia Rincon, L., Simas, H., Martins, D. "Wrench distribution of a cooperative robotic system using a modified scaling factor method", Journal of the Brazilian Society of Mechanical Sciences and Engineering, 40(4), Article Number: 177, 2018. https://doi.org/10.1007/s40430-018-1090-z

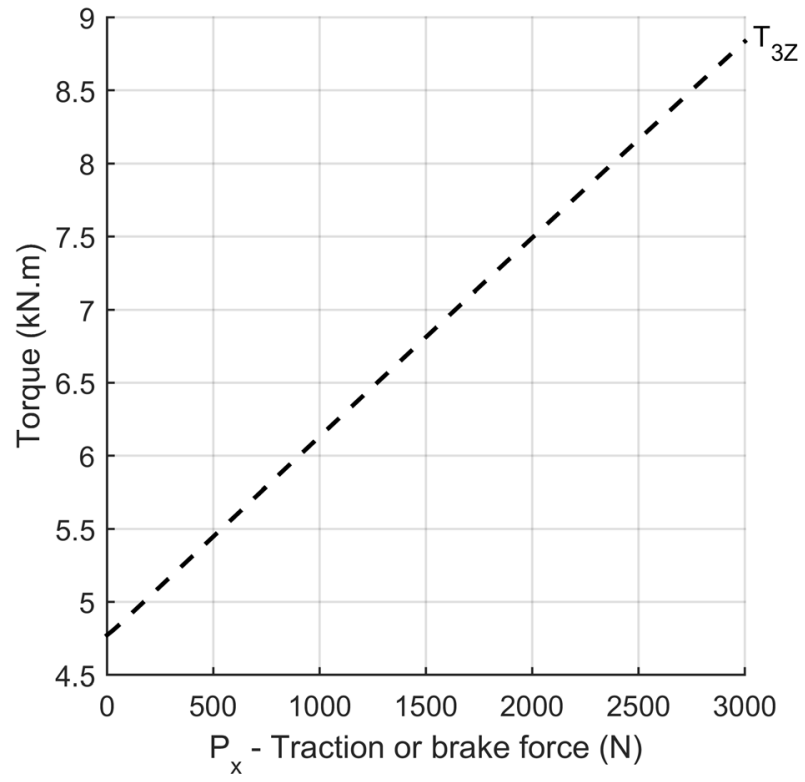

Fig. 7 Torque.

external forces displaced, causing a significant increase in the internal forces that act upon the mechanism. This allows for better decision-making when designing and selecting the analyzed mechanisms' elements and equipment.

[4] Frantz, J. C., Mejia Rincon, L., Simas, H., Martins, D. "Analysis of wrench capability for cooperative robotic systems", In: 23rd $\mathrm{ABCM}$ International Congress of Mechanical Engineering (COBEM 2015), Rio de Janeiro, Brazil, 2015, Article Number: 0838. https://doi.org/10.13140/RG.2.1.1673.0965

[5] Papadopoulos, E., Gonthier, Y. "On manipulator posture planning for large force tasks", In: Proceedings of 1995 IEEE International Conference on Robotics and Automation, Nagoya, Japan, 1995, pp. 126-131.

https://doi.org/10.1109/ROBOT.1995.525274 
[6] Moreno, G., Nicolazzi, L. C., De Souza Vieira, R., Martins, D. "Stability of long combination vehicles", International Journal of Heavy Vehicle Systems (IJHVS), 25(1), pp. 113-131, 2018. https://doi.org/10.1504/IJHVS.2018.089897

[7] Erthal, J. L., Nicolazzi, L. C., Martins, D. "Kinematic analysis of automotive suspensions using Davies' method", In: 19th International Congress of Mechanical Engineering (COBEM 2007), Brasilia, Brazil, 2007, Article Number: 423.

[8] Moreno, G., Serrano, J. C., Espinel Blanco, E. "Análisis de Técnicas que Permitan Incrementar la Estabildad de Carrotanques" (Analysis of Techniques that Allow to Increase the Stability of Road Tankers), Revista Colombiana de Tecnologías de Avanzada, 1(35), pp. 52-56, 2020. [online] Available at: http://www.unipamplona.edu.co/unipamplona/portalIG/home_40/recursos/revistas/17022020/revista_35. jsp [Accessed: 06 April 2020] (in Spanish)

[9] Frantz, J. C., Mejia Rincon, L., Simas, H., Martins, D. "A New Methodology for the Balancing of Mechanisms Using the Davies' Method", In: International Symposiu on Multibody Systems and Mechatronics, Florianópolis, Brazil, 2018, pp. 203-212. https://doi.org/10.1007/978-3-319-67567-1_19

[10] Davies, T. H. "The 1887 committee meets again. Subject: freedom and constraint", In: Proceedings of A Symposium Commemorating the Legacy, Works, and Life of Sir Robert Stawell Ball, Upon the 100th Anniversary of 'A Treatise on the Theory of Screws', Cambridge, UK, pp. 1-56, 2000.

[11] Cazangi, H. R. "Aplicação do Método de Davies para Análise Cinemática e Estática de Mecanismos com Múltiplos Graus de Liberdade" (Application of the Davies Method for Kinematic and Static Analysis of Mechanisms with Multiple Degrees of Freedom), Master's Thesis, Federal University of Santa Catarina, 2008. (in Spanish)

[12] Tsai, L. W. "Mechanism Design: Enumeration of Kinematic Structures According to Function", CRC Press, Boca Raton, FL, USA, 2001
[13] Cazangi, H. R., Martins, D. "Kinematic analysis of automotive gearbox mechanisms using Davies' method", In: 19th International Congress of Mechanical Engineering (COBEM, 2007), Brasilia, Brazil, 2007, Article Number: 475.

[14] Myszka, D. H. "Machines \& Mechanisms: Applied Kinematic Analysis", Pearson Prentice Hall, Boston, MA, USA, 2012.

[15] Moreno, G., Frantz, J. C., Nicolazzi, L. C., Vieira, R. S., Martins, D. "Stiffness and Deformation of Mechanisms with Locally Flexible Bodies: A General Method Using Expanded Passive Joints", In: 16th International Symposium on Advances in Robot Kinematics, Bologna, Italy, 2018, pp. 285-292. https://doi.org/10.1007/978-3-319-93188-3_33

[16] Davies, T. H. "Coupling, coupling networks and their graphs", Mechanism and Machine Theory, 30(7), pp. 991-1000, 1995. https://doi.org/10.1016/0094-114X(95)00023-R

[17] Farias Dos Santos, C. H., Guenther, R., Martins, D., De Pieri, E. R. "Inverse Kinematics of the Underwater Vehicle-Manipulator Systems Using Kinematic Constrains", In: 11th IEEE International Conference on Methods and Models in Automation and Robotics, Miedzyzdroje, Poland, 2005, pp. 573-578.

[18] Davies, T. H. "Mechanical Networks - I Passivity and redundancy", Mechanism and Machine Theory, 18(2), pp. 95-101, 1983. https://doi.org/10.1016/0094-114X(83)90100-3

[19] Davies, T. H. "Freedom and Constraint in Coupling Networks", Proceedings of the Institution of Mechanical Engineers, Part C: Journal of Mechanical Engineering Science, 220(7), pp. 989-1010, 2006. https://doi.org/10.1243/09544062C09105

[20] Shukla, G., Whitney, D. E. "The path method for analyzing mobility and constraint of mechanisms and assemblies", IEEE Transactions on Automation Science and Engineering, 2(2), pp. 184-192, 2005. https://doi.org/10.1109/TASE.2005.844120 


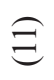

$$
\begin{aligned}
& \text { R. } \\
& R^{*}-1-00001-1 \\
& H^{m} 0000-0000 \\
& \text { IN } 00000000 \\
& \text { I } \\
& \text { L } \\
& x^{\tilde{N}} 000000-000 \\
& \text { 庄胥 } 000-00000 \\
& x^{N} 0-0000000 \\
& x^{2}-T T T \neg 000 \\
& x^{5}--T \uparrow T \uparrow 000 \\
& \stackrel{\Omega}{\because} 0000000000 \text { - } \\
& \text { I } 00000000001 \\
& x^{2} 00000000-0 \\
& x^{5} 0000000010 \\
& \sum^{\infty} 000000-00 \\
& x^{\infty} 0000000-00 \\
& \sum 00000-000 \\
& \text { L } \\
& x^{n} 0000-0000 \\
& x^{n} 0000-0000 \\
& z^{7} 000-00000 \\
& L^{2} 000-00000 \\
& \text { I } \mathrm{r}^{\mathrm{m}} 0-000000 \\
& x^{m} 00-000000 \\
& \text { ño- } \\
& \text { IN } 0 \text { - } 0000000 \\
& L^{2}-00000000
\end{aligned}
$$

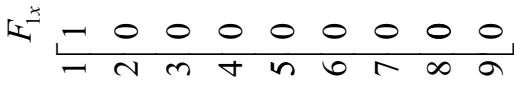

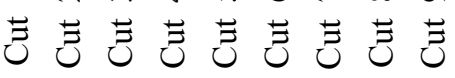

